\title{
Breast and axillary surgery in malignant breast disease: a review focused on literature of 2018 and 2019
}

\author{
Nina Ditsch ${ }^{\mathrm{a}}$, Isabel T. Rubio ${ }^{\mathrm{b}}$, Maria L. Gasparric, Jana de Boniface ${ }^{\mathrm{d}, \mathrm{e}}$ \\ Thorsten Kuehn ${ }^{\dagger}$
}

\begin{abstract}
Purpose of review
There have been fundamental changes in the surgical approach to breast cancer management over the last decades. The primary objective of achieving locoregional control, however, remains unchanged.

\section{Recent findings}

In addition to strategies optimizing systemic treatment and radiotherapy, current discussions focus on improving the surgical approach to breast cancer. Especially in view of the increasingly pivotal role of neoadjuvant chemotherapy NAT/NAC (NACT), gauging the extent of tissue removal in the breast and the width of resection margins in breast-conserving surgery is highly important, as is the extent of axillary surgery. Although sentinel lymph node (SLN)-positive patients always underwent axillary lymph node dissection in the past, this paradigm has been challenged in recent years. Targeted axillary dissection (TAD) has emerged as a new staging option in biopsy-proven node-positive patients who convert to clinical node negativity ( $\mathrm{CNO}$ ) after NACT. TAD combines the removal of the SLN and of the target lymph node marked prior to NACT. The accuracy of axillary staging both before and after NACT plays an important role for prognostication and multidisciplinary treatment plans, while its extent has significant effects on patients' arm morbidity and quality of life.
\end{abstract}

\section{Summary \\ The current review focuses on recent evidence regarding surgical management of the breast and axilla in patients with primary breast cancer based on a PubMed and EMBASE literature search for publication years 2018 and 2019.}

\section{Keywords}

breast-conserving surgery, mastectomy, neoadjuvant chemotherapy, sentinel lymph node, targeted axillary dissection

\section{INTRODUCTION}

Surgery is a crucial part of the multimodal therapy concept. Current surgical strategies in breast cancer management primarily focus on reducing the extent of tissue removal in breast and axilla to improve patients' quality of life as well as the esthetic outcome while maintaining high oncological safety. With the introduction of the sentinel lymph node biopsy (SLNB) for patients with clinically node-negative breast cancer, axillary lymph node dissection (ALND) was replaced as a staging procedure for patients undergoing primary surgery. Based on affirmative data regarding detection and false negative rates, the removal of the SLN is now also recommended after neoadjuvant chemotherapy NAT/NAC (NACT) in patients with initially clinical negative nodes [1-5]. There has been an increase in detection of isolated tumor cells [pNO(i+)] and micrometastases (pN1mi) on axillary staging after NACT [6]. The prognostic impact of low-volume nodal involvement and the role of locoregional therapy in this situation,

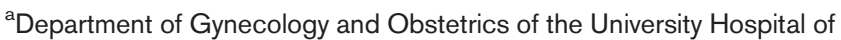
Augsburg, Augsburg, Germany, ${ }^{b}$ Breast Surgical Oncology Unit, Clinica Universidad de Navarra, Madrid, Spain, 'Breast Surgery Unit, San Raffaele University Hospital, Milan, Italy, ${ }^{\mathrm{d} D e p a r t m e n t}$ of Molecular Medi-

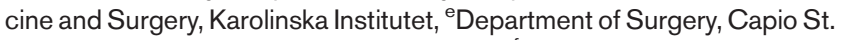
Göran's Hospital, Stockholm, Sweden and 'Department of Gynecology and Obstetrics, Interdisciplinary Breast Center, Esslingen, Germany

Correspondence to Nina Ditsch, Department of Gynecology and Obstetrics of the University Hospital of Augsburg, Stenglinstr. 2, 86156 Augsburg, Germany. Tel: +49 821 4002331;

e-mail: nina.ditsch@uk-augsburg.de
} 


\section{KEY POINTS}

- Margin status is still the most important prognostic factor for local control after primary surgery.

- SLN biopsy (SLNB) is the standard of care staging procedure in $\mathrm{cNO}$ patients with invasive disease.

- Axillary lymph node dissection after a positive SLNB may be avoided in $\mathrm{cNO}$ patients with micrometastasis or macrometastasis in up to two SLNs.

- Combined methods of SLNB and removal of axillary metastases marked pre-NACT (TAD) achieve considerably better FNRs than SLNB alone.

- $T A D$ is considered as the preferred procedure in patients who convert from $\mathrm{cN} 1$ to $\mathrm{ycNO}$.

however, are still unclear. An additional unanswered question concerns those patients with an initially clinically positive axilla $(\mathrm{cN}+)$ who show a complete remission after NACT: how far can de-escalation of axillary surgery proceed, and when can ALND be avoided? In view of new strategies for post-NACT treatment options for patients with residual disease $[7,8]$, the diagnostic accuracy of surgical breast (ypT) and lymph node (ypN) staging has gained even more significance even in minimal residual disease.

The current review summarizes recently published evidence (focusing on the last 2 years) on the extent of surgery to the breast and the axilla both in primary surgery and after NACT, with special regard to tumor biology, locoregional radiotherapy, and systemic therapy.

\section{REVIEW}

\section{Surgery of the breast: as primary therapeutic approach}

Margin status is still the most important prognostic factor for local control after primary surgery. No ink on tumor for invasive carcinomas and a $2 \mathrm{~mm}$ margin for ductal carcinoma in situ are considered sufficient if combined with whole-breast irradiation [9]. For both breast-conserving surgery (BCS) and mastectomy, a main objective remains the best possible aestheticreconstructive outcome in combination with highest oncological safety. Studies on patient satisfaction report rates above $80 \%$ for all reconstructive procedures [10] which could possibly be increased with improved methods (e.g. fat supplementation). There is uncertainty regarding the preferred method but a clear relation between the surgical outcome and the education and skills of the physician and different breast cancer centers has been shown [11].

\section{Surgery of the breast: after neoadjuvant chemotherapy}

Recent Early Breast Cancer Trialists Collaborative Group data showed a 15 year local recurrence rate higher for patients who underwent NACT (21.4\%) than for those receiving systemic therapy after primary surgery (15.9\%) [12]. Despite this, no prognostic relevance for disease-free survival (DFS) and overall survival was observed. Importantly, a large number of patients did not undergo any breast surgery but only radiotherapy after NACT; for those patients, the increase in local recurrence rates was $11.3 \%$ compared with patients not receiving NACT, while local recurrence rates in patients who were operated after NACT were only increased by 3.2. In the meantime, imaging modalities and radiotherapy approaches have significantly improved. Therefore, these data can hardly be used to adequately define the necessary extent of surgery to the tumor bed. Instead, a recently published retrospective analysis including 382 patients treated with NACT between 2002 and 2014 reported an overall local recurrence rate of only $3.9 \%$ after 5 years. Even in patients with a negative margin width of $1 \mathrm{~mm}$ or less $(n=65 / 382,17.0 \%)$, no local recurrence was observed. The authors concluded that a policy of 'no tumor on ink' was sufficiently effective for the resection of the tumor after NACT [13].

In the Responder trial, the role of vacuumassisted biopsy (VAB) to predict a pathological complete response (pCR) was compared with excisional biopsy. Although the trial was based on the hypothesis that breast surgery might be omitted in patients with a good response to chemotherapy, it had to be discontinued due to an excessively high false negative rate (FNR) [17.8\%, 95\% confidence interval (CI): $12.8-23.7 \%]$ in the $\mathrm{VAB}$ group.

\section{Surgery of the breast: reconstruction methods}

When mastectomy is indicated, skin-and nipplesparing techniques with immediate reconstruction in selected patients are associated with no increase in recurrence rates yet a better quality of life compared with conventional mastectomy and delayed reconstruction. Depending on the location of the tumor, the nipple-areola complex can be preserved. Following a mastectomy, reconstruction can be carried out using implants or autologous tissue or a combination of both [14]. For single use of implants combinations with acellular dermal matrices (ADM) or meshes are the current standard of care. One-stage implantbased breast reconstruction with ADM does not yield superior results in terms of patient-reported quality of life compared with two-stage reconstruction [15] but current data (with 6-months follow-up) showed an 
association with more adverse outcomes requiring surgical intervention in contrast to conventional implant-based reconstruction without ADM [16].

\section{Surgery of the axilla: as first therapeutic approach}

The surgical approach toward the axilla has been a controversial issue over the last decades. Data from recently published trials have provided practicechanging recommendations for this scenario. However, further controversies have been triggered in the surgical community, resulting in widely differing recommendations [17].

SLN biopsy has replaced ALND in clinically nodenegative breast cancer patients who undergo primary surgery, with false negative rates below 10\%. Since adjuvant systemic treatment decisions are mainly based on tumor biology, the question whether axillary staging can be omitted altogether has become a subject of discussion. Two trials addressing this issue have recently finished accrual (INSEMA, SOUND). The AMAROS [18], The American College of Surgeons Oncology Group (ACOSOG) Z0011 [19] and IBCSG 23-01 studies provided evidence that ALND can be omitted in patients with occult SLN involvement or be replaced by locoregional radiotherapy. As a result of these trials, whose methodological limitations are well known, ALND is being increasingly abandoned in clinical routine: A big data analysis of 1131363 women with clinical stage I-III breast cancer in the US National Cancer Data Base (255306 with ALND and 876057 without ALND) showed declining ALND rates from $2004(32 \%)$ to $2014(16 \% ; P<0.001)$ in both BCS and mastectomy patients [20]. After adjustment, this effect was maintained, with ALND rates decreasing with each additional year (odds ratio $=0.90,95 \%$ CI $0.89-0.90$ ). Resident procedure volumes similarly declined from 1999 to 2017 $(P<0.001)$, indicating that less experienced junior residents who are predominantly performing ALND do so with very low absolute numbers. Similarly, a Dutch study observed a reduction of the ALND rate from $75 \%$ in 2011 to $17 \%$ in 2015 [21"]. Important contributing factors to performing an ALND were unfavorable tumor morphology or biology. The extent of axillary surgery correlates with the surgeon's experience: Those with more experience perform less radical procedures than less experienced surgeons [22"']. Furthermore, ALND rates vary considerably between different institutions [23,24"']. A German study group analyzed 188909 data records and identified 13741 patients with cT1/2 NOM0 cancers with one to two positive SLNs, treated with BCS and tangential field irradiation. In 2008, an ALND was performed in $94.6 \%$ of these cases, while in 2015 , this figure had decreased to only $46.9 \%$. Similar trends were reported by the EUSOMA working group [24"']: Analyzing 671 patients fulfilling the criteria of the Z0011 trial, rates of ALND showed a statistically significant decrease from 2010 (89\%) to 2011 (73\%), reaching $46 \%$ in $2016(P<0.001)$.

\section{Surgery of the axilla: after neoadjuvant chemotherapy}

SLN detection rates are very high (97.6\%) after NACT in patients who initially present with a clinically negative lymph node status, and the omission of ALND after a negative SLN biopsy is associated with a negligibly small number of axillary recurrences as shown in the GANEA-2 (GAnglion sentinelle après chimiothérapie NÉoAdjuvanteMARI - axillary lymph node marking with radioactive iodine seeds) trial, which was first to address the safety of SLN biopsy in this setting. In 419 enrolled patients, only one axillary recurrence $(0.2 \%)$ was reported after a median follow-up of 3 years, along with a 3-year event-free survival of $97.8 \%$ [25"']. This is similar to retrospective data from the MD Anderson Cancer Center, which reported a $1.2 \%$ regional recurrence rate after a median follow-up of 47 months in patients having a negative SLN biopsy after NACT compared with $0.9 \%$ for those having a negative SLN biopsy upfront [1].

In the case of cN1, the prospective SENTINA (SENTinel NeoAdjuvant) and ACOSOG Z1071 trials showed FNRs higher than 10\%. In the Canadian SN FNAC trial, the FNR was $8.4 \%$ in ypNO, but increased to $13.3 \%$ if ITCs were classified as node negative. FNR was reduced when immuno-histochemical was performed or ultrasound was added. In case of postNACT axillary ultrasound FNR decreased from 8.4 to $2.7 \%$ [26"]. Little data are currently available to determine the necessity of ALND in cN+ and histopathological complete remission of SLN (ypNO). Only two retrospective studies are available that show no benefit of an additional ALND in cN+ypNO patients compared with SLN biopsy alone $[27,28]$. Importantly, post-NACT ultrasound assessment of the axilla has a very low sensitivity and cannot replace SLN biopsy as a staging procedure [29].

A hypothesis-generating finding from ACOSOG Z1071 suggested that the marker localization of a preoperatively positive lymph node by clip or radioactive seed and its targeted removal after NACT (TLNB-targeted lymph node biopsy) could reduce the FNR to $10.1 \%$ (Fig. 1). For the localization of the targeted lymph node (TLN), several techniques such as placement of clips, coils, radioactive seeds, and carbon tattooing have been described [30-32, $\left.33^{-\prime}, 34,35\right]$. In a retrospective analysis of a prospective database, the FNR could be further reduced to $2 \%$ if 


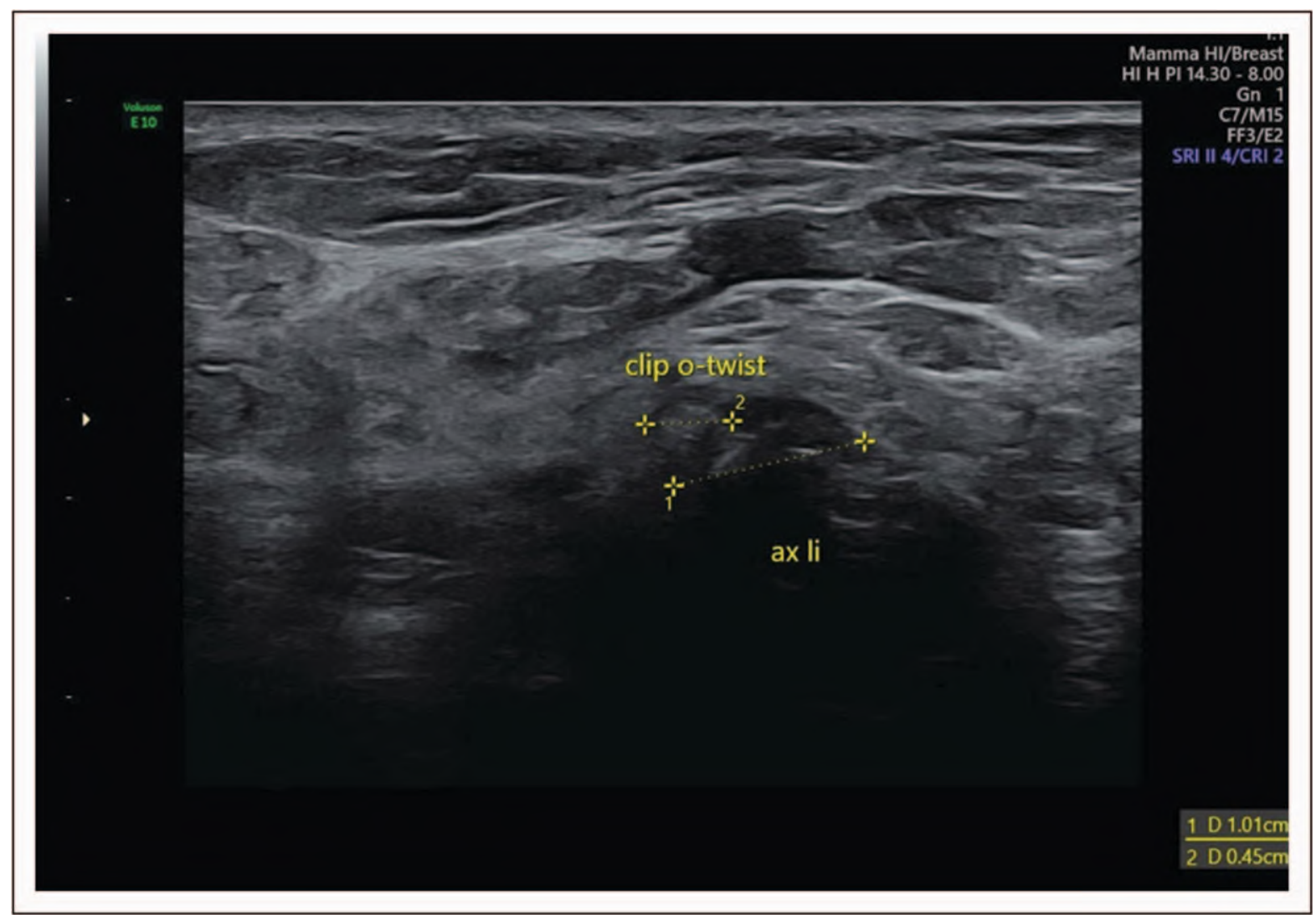

FIGURE 1. Sonographic-guided marker localization of a preoperatively positive axillary lymph node by clip.

both SLN and TLN were removed [36]. This combination procedure, named targeted axillary dissection (TAD), was confirmed to achieve the lowest FNRs in a recent meta-analysis [37"'] including 20 studies with 2217 patients: 17 studies on SLN biopsy, one on MARI (marking of the tumor-positive lymph node with a radioacctive iodine seed) and two on combination procedures. Overall, the axillary pCR rate was $37 \%$. For SLN biopsy alone, pooled rates of detection rate and FNR were 89 and $17 \%$, respectively, and the negative predictive value (NPV) ranged from 57 to $86 \%$. For MARI, the detection rate was $97 \%$, FNR $7 \%$, and the NPV 83\%: In the MARI procedure, predominantly used in The Netherlands, a radioactive seed is placed in the affected lymph node at biopsy and left in place throughout NACT. The combination of MARI with a SLN biopsy is assessed in the ongoing RISAS trial, thus implementing TAD [38]. For combination procedures, detection rate was $100 \%$, FNRs ranged from 2 to $4 \%$ and the NPV from 92 to $97 \%$. Table 1 gives an overview on recently published trials that compare different minimally invasive staging procedures and their combination with regard to feasibility (detection rate) and accuracy to predict the axillary status (FNR). These studies show clear advantages for the combination procedures (TAD) as compared with SLN biopsy or TLNB alone.
The TAD identification rate still needs improvement since the optimal localization technique is not yet clear. The most common method, that is clip localization of the TLN, is not yet a reproducible procedure for clinical routine. High failure rates of up to $30 \%$ have been reported in combination with preoperative wire localization [53"]. A significantly lower failure rate of $17 \%$ was achieved with ultrasound-guided TLN detection [54]. TAD seems best suited to reduce the extent of axillary surgery with good clinical response of NACT, even though prospective data on significantly larger collectives will yet have to confirm this. Results from ongoing trials are expected in the near future (SENTA, PreATNEC, GANEA 3, TATTOO). So far, however, no data regarding oncologic safety (recurrence rates) and morbidity are available that compare ALND, SLNB, TLNB, and TAD. A Europe-wide prospective register (AXSANA) is currently planned by the EUBREAST study group to address these issues.

\section{Surgery of the axilla: individual subgroup analyses}

A study from the American National Cancer Data Base (NCDB) included 30821 patients with cT1/cT2 
Table 1. Selection of the most important and current studies on sentinel lymph node biopsy, single removal of marked $L N$ and combination methods (targeted axillary dissection) of preneoadjuvant chemotherapy histopathologically confirmed axillary lymph nodes

\begin{tabular}{|c|c|c|c|c|c|}
\hline Author & Method & $n$ & Markers & $\begin{array}{l}\text { Identification rate } \\
(\%)\end{array}$ & FNR $(\%)$ \\
\hline Alvarado et al. [39] & SLNB & 150 & Tc and/or blue & 92.7 & $21(12-32)$ \\
\hline Boileav et al. [40] & SLNB & 153 & Tc and/or blue & 87.8 & $8(3-17)$ \\
\hline Boughey et al. [41] & SLNB (ACOSOG Z1071) & 689 & Tc and/or blue & 92.7 & $15(11-19)$ \\
\hline Enokido et al. [42] & SLNB & 143 & Tc and/or blue & 90.9 & $21(12-33)$ \\
\hline Kuehn et al. [43] & SLNB & 592 (Arm C) & Tc with/without blue & 82.6 & $19(10-31)$ \\
\hline Park et al. [35] & SLNB & 169 & Single radioactive & 94.9 & $22(14-31)$ \\
\hline Pinero-Madrona et al. [44] & SLNB & 94 & Tc with/without blue & 84.0 & $40(21-61)$ \\
\hline Yu et al. [45] & SLNB & 122 & Single blue & 95.8 & $36(17-59)$ \\
\hline Zetterlund et al. [46] & SLNB & 195 & Tc and/or blue & 77.9 & $14(8-23)$ \\
\hline Donker et al. [47] & $\begin{array}{l}\text { Marking of }+\mathrm{LN} \text { (with } \\
\text { radioactive lodinel seed; } \\
\text { MARI) }\end{array}$ & 100 & $\begin{array}{l}\text { Preiodine seed } \\
\text { Post-NA }\end{array}$ & 97.0 & $7(2-16)$ \\
\hline Dashevsky et al. [48] & Marked $+\mathrm{LN}$ & 28 & $\begin{array}{l}\text { Preclip postultrasound } \\
\text { guided wire localization }\end{array}$ & - & n.d. \\
\hline Kim et al. [49"] & Marked $+\mathrm{LN}, \mathrm{SLN}$ & & $\begin{array}{l}\text { Preimage-guided clip } \\
\text { Postultrasound }\end{array}$ & 95.8 & - \\
\hline Nguyen et al. [50] & Marked LN+, SLN & 56 & 125I Radioactive seed & $\begin{array}{l}72 \text { (with only } \\
\text { ultrasound); } 91 \text { in } \\
\text { combination with CT, } \\
\text { palpation and SLNB }\end{array}$ & - \\
\hline Park et al. [31] & Marked LN+; SLNB, ALN & 20 & Charcoal tattooing & 80.0 & 20 (combined 0) \\
\hline Hartmann et al. [34] & TLN, SLNB, ALND & 30 & $\begin{array}{l}\text { Preclip } \\
\text { Postultrasound-guided wire } \\
\quad \text { localization }\end{array}$ & $\begin{array}{l}70.8 \\
\text { Clipped node } \\
\text { identification rate }\end{array}$ & - \\
\hline Plecha et al. [51] (retrospective) & Marked LN+, SLNB, ALND & 73 & Clip & 97 & - \\
\hline Caudle et al. [36] & $\begin{array}{l}\text { TAD } \\
\text { SLNB }+125 \text { I seed placement } \\
\text { in clipped node after NACT } \\
\text { completion }\end{array}$ & & $\begin{array}{l}\text { Preclip } \\
\text { Postiodine seed (SLN: Tc } \\
\text { and/or blue) }\end{array}$ & 100.0 & $2(0-11)$ \\
\hline Siso et al. [33""] & $\begin{array}{l}\text { Intraoperative ultrasound- } \\
\text { guided excision of axillary } \\
\text { clip }\end{array}$ & 46 & $\begin{array}{l}\text { Preclip } \\
\text { Post-NA (SLN: Tc and/or } \\
\text { blue) }\end{array}$ & n.d. & $4.1(0-21)$ \\
\hline Boughley et al. [52] & Marked LN+, SLNB, ALND & 203 & Clip & 83 & $\begin{array}{c}6.8(1.9-16.5) \\
\text { clipped node within } \\
\text { SLN; } \\
19(5.4-41.9) \text { clipped } \\
\text { node in ALND }\end{array}$ \\
\hline Van Ninjatten et al. [38] & $\begin{array}{l}\text { SLNB }+125 I \text { seed placement } \\
\text { before NAC (RISAS) }\end{array}$ & 225 & lodine-125 seed & - & - \\
\hline
\end{tabular}

Patient numbers for only SLNB under $n=100$ were excluded in SLNB-focused studies. Studies for TLN, MARI and TAD included lower patient numbers because of the absence of statistical valid data. ALN, axillary lymph node; ALND, axillary lymph node dissection; CT, computed tomography; FNR, false negative rate; NACT, neoadjuvant chemotherapy; SLN, sentinel lymph node; Tc, technetium; SLNB, sentinel lymph node biopsy; TAD, targeted axillary dissection; TLN, target lymph node.

cNO/N1 breast cancer treated with NACT. PostNACT surgical staging of the axilla demonstrated a $1.6 \%$ rate of axillary node positivity in initially cNO patients with HER2-positive or triple-negative breast cancer who achieved a breast pCR [55"']. This is in clear contrast to the rate of axillary involvement in the initially cNO group not achieving breast pCR with $16.9 \%$ in HER2-positive and $12.6 \%$ in triplenegative cases, and for the initially cN1 group (Table 2). The authors hypothesize that axillary surgery could be entirely avoided in these selected cases. In the group of hormone receptor-positive
HER2-negative breast carcinomas, a correlation between breast pCR and ypNO status was found to a lesser degree: For initially cNO patients with a breast pCR, the ypN1 rate was $4 \%$. These results confirm previous studies with smaller patient numbers which showed a correlation between breast and axillary pCR and their association with intrinsic subtypes [56,57"]. In contrast to triple-negative and HER2-positive tumors conversion from $\mathrm{cN} 1$ to ypNO was significantly lower for estrogen receptor $+/$ Her2 (ERBB2) - tumors $(50$ versus $31.9 \%$ ) (Table 3; [57"]). 
Table 2. Relationship between pathological complete response and axillary lymph node involvement after neoadjuvant chemotherapy

\begin{tabular}{|c|c|c|c|c|}
\hline \multirow[b]{2}{*}{ Clinical node status } & \multirow[b]{2}{*}{ No of pts } & \multicolumn{3}{|c|}{ No. (\%) of patients } \\
\hline & & ypNO & ypN1 & ypN2/ypN3 \\
\hline \multicolumn{5}{|l|}{ Breast $p C R$} \\
\hline \multicolumn{5}{|c|}{ Biologic subtype of $\mathrm{cNO}$ disease } \\
\hline HR-positive/Her2-positive & 1732 & 1696 (97.9) & $N R(<3)$ & $N R(<1)$ \\
\hline vHR-negativeHer2-positive & 1330 & $1317(99.0)$ & $13(1.0)$ & 0 \\
\hline TNBC & 2315 & $2279(98.4)$ & $N R(<2)$ & $N R(<1)$ \\
\hline HR-positive/Her2-negative & 646 & $620(96.0)$ & $26(4.0)$ & 0 \\
\hline \multicolumn{5}{|c|}{ Biologic subtype of $\mathrm{cNI}$ disease } \\
\hline HR-positive/Her2-positive & 959 & $831(86.7)$ & $110(11.5)$ & $18(1.9)$ \\
\hline HR-negative/Her2-positive & 842 & 747 (88.7) & $N R(<11)$ & $N R(<2)$ \\
\hline TNBC & 1229 & 1056 (85.9) & $150(12.2)$ & $23(1.9)$ \\
\hline HR-positive/Her2-negative & 711 & $494(69.5)$ & $187(26.3)$ & $30(4.2)$ \\
\hline \multicolumn{5}{|l|}{ Residual breast disease } \\
\hline \multicolumn{5}{|c|}{ Biologic subtype of cNO disease } \\
\hline HR-positive/Her2-positive & 2870 & $2336(81.4)$ & $475(16.6)$ & $59(2.1)$ \\
\hline HR-negative/Her2-positive & 870 & $772(88.7)$ & $82(9.4)$ & $16(1.8)$ \\
\hline TNBC & 3907 & $3415(87.4)$ & 427 (10.9) & 65 (1.7) \\
\hline HR-positive/Her2-negative & 4423 & 2959 (66.9) & $1203(27.2)$ & $261(5.9)$ \\
\hline \multicolumn{5}{|c|}{ Biologic subtype of $\mathrm{cN} 1$ disease } \\
\hline HR-positive/Her2-positive & 1672 & $581(34.7)$ & $858(51.3)$ & $233(13.9)$ \\
\hline HR-negative/Her2-positive & 691 & $308(44.6)$ & $299(43.3)$ & $84(12.2)$ \\
\hline TNBC & 2064 & 737 (35.7) & $932(45.2)$ & $395(19.1)$ \\
\hline HR-positive/Her2-negative & 4560 & 785 (17.2) & $2487(54.5)$ & $1288(28.2)$ \\
\hline
\end{tabular}

Extent of nodal disease at surgery by pathologic nodal category. $H R$, hormone receptor; $\mathrm{pCR}$, pathological complete response. TNBC, triple negative breast cancer. Adapted from [55" $]$.

Table 3. Comparison of different types of breast cancer with respect to response to neoadjuvant chemotherapy in case of ypNO

\begin{tabular}{|c|c|c|c|}
\hline & Breast $p C R$ and ypNO & No breast $p C R$ and ypNO & P value* \\
\hline $\begin{array}{l}\text { cT1-3 No } \\
\mathrm{ER}+\mathrm{Her} 2+ \\
n=277\end{array}$ & $98.4(122 / 124)$ & $85.0(130 / 153)$ & $P<0.001$ \\
\hline $\begin{array}{l}c T 1-3 \mathrm{N1} \\
\mathrm{ER}+\operatorname{Her} 2+ \\
n=308\end{array}$ & $51.8(58 / 112)$ & $19.9(39 / 196)$ & $P<0.001$ \\
\hline $\begin{array}{l}\text { cTl } 1-3 \mathrm{NO} \\
\mathrm{ER}-\mathrm{Her} 2+ \\
n=128\end{array}$ & $100(97 / 97)$ & $90.3(28 / 31)$ & $P=0.013$ \\
\hline $\begin{array}{l}\text { cT1 } 1-3 \mathrm{N1} \\
\mathrm{ER}-\mathrm{Her} 2+ \\
n=233\end{array}$ & $46.7(70 / 150)$ & $30.1(25 / 83)$ & $P=0.014$ \\
\hline $\begin{array}{l}\mathrm{cT} 1-3 \mathrm{NO} \\
\mathrm{ER}+\mathrm{Her} 2- \\
n=932\end{array}$ & $93.3(83 / 89)$ & $64.7(545 / 843)$ & $P<0.001$ \\
\hline $\begin{array}{l}\mathrm{CT} 1-3 \mathrm{~N} 1 \\
\mathrm{ER}+\mathrm{Her} 2- \\
n=1.404\end{array}$ & $31.9(38 / 119)$ & $5.1(65 / 1285)$ & $P<0.001$ \\
\hline $\begin{array}{l}c T 1-3 \text { No } \\
\text { triple negative } n=337\end{array}$ & $98.5(139 / 132)$ & $87.3(179 / 205)$ & $P<0.001$ \\
\hline $\begin{array}{l}\text { CT1 }-3 \mathrm{N1} \\
\text { triple negative } \\
n=465\end{array}$ & $48.5(79 / 163)$ & $15.6(47 / 302)$ & $P<0.001$ \\
\hline
\end{tabular}

Data are presented as percentages with the numbers in parentheses. $\chi^{2}$ test and Fisher exact test between pts with breast $\mathrm{pCR}$ and ypNO versus pts without breast pCR and ypNO. Er indicates estrogen receptor, triple negative: negative for ER, PR and Her2. Overview of $y p N O$ in the case of breast $p C R$ and without breast $p C R$ after NACT for the different breast cancer subtypes. ER, estrogen receptor; NACT, neoadjuvant chemotherapy; pCR, pathological complete response. Adapted from [57"] ${ }^{*} P<0.05$ value with significance. 


\section{CONCLUSION}

The development of clinical guidelines for the management of the axilla in breast cancer is a work in progress. Despite the known methodological shortcomings of the ACOSOG Z0011 trial which explains the cautious implementation of its results in current guidelines, ALND is increasingly being abandoned in daily routine for patients with clinically occult involvement of the SLN even if the criteria of the underlying trials (ACOSOG Z0011, IBCSG 23-01, AMAROS) are not fulfilled.

After NACT, TAD has been proposed to replace conventional ALND with its concomitant morbidity for patients who convert from a $\mathrm{cN} 1$ to a $\mathrm{ycNO}$ status.

Based on the existing data, there are still no uniform guidelines for axillary surgery in Europe and worldwide. The observed correlation of breast and axillary pCR after NACT and its association with the intrinsic subtype may further de-escalate surgical management in the axilla.

Taking into account the data reported in this article, the following points are regarded as trendsetting for the current surgical treatment of breast and axilla.

(1) No ink on tumor is the accepted standard for invasive resection margins for patients who undergo primary BCS.

(2) After NACT, there is no reason to aim for wider resection margins than in primary surgery.

(3) SLN biopsy is the standard of care staging procedure in $\mathrm{CNO}$ patients with invasive disease.

(4) ALND after a positive SLN biopsy may be avoided in cNO patients with micrometastasis or macrometastasis in up to two SLNs,

(5) For the following statement no evidence is available, but after discussion the German Guidelines state that patients fulfilling the above criteria who undergo mastectomy, may also avoid axillary dissection after multidisciplinary discussion of individual cases and consideration of axillary irradiation.

(6) Suspicious lymph nodes should be assessed by core needle biopsy.

(7) Combined methods of SLN biopsy and removal of axillary metastases marked pre-NACT (TAD) achieve considerably better FNRs than SLN biopsy alone, and are therefore considered as the preferred procedure in patients who convert from cN1 to ycNO.

(8) The ideal localization method for TAD has yet to be defined.

(9) Further studies are needed to assess the prognostic significance of axillary surgery on DFS and overall survival, and provide information on the need for radiotherapy after NACT in case of axillary pCR.

Results from large, confirmatory randomized trials that assess the omission of ALND in case of a positive SLN biopsy are expected in the near future (INSEMA, POSNOC, SENOMAC, SINODAR-ONE).

In view of the ongoing reduction of the extent of surgery in the axilla, the question arises whether radiotherapy of the axilla needs to be performed, and if yes, what target volume should be irradiated. Further trials will show whether a good response to NACT may further de-escalate locoregional treatment (Alliance 011202 and NSABP-51/RTOG 1304).

In summary, this review is limited by the heterogeneity of included studies with small patient numbers and different patient populations. Thus, results and consequences should be interpreted with caution and could be subject to change in the near future.

\section{Acknowledgements}

J.d.B. is supported by the Junior Clinical Investigator Award granted by the Swedish Cancer Foundation. Ongoing studies: Alliance 11202 (NCT01901094. Comparison of Axillary Lymph Node Dissection With Axillary Radiation for Patients With Node-Positive Breast Cancer Treated With Chemotherapy. Principal Investigator: Judy Boughey. Mayo Clinic. Available at: https:// clinicaltrials.gov/ct2/show/NCT01901094 Accessed 4 February 2018; NCT01872975).

GANEA3 (Institut Cancerologie de l'Ouest. Sentinel lymph node after neoadjuvant chemotherapy in breast carcinoma (GANEA-3) [NCT03630913]. www.clinicaltrials.gov. Accessed 12 July 2019). NSABP-51/RTOG 1304 (Standard or Comprehensive Radiation Therapy in Treating Patients With Early-Stage Breast Cancer Previously Treated With Chemotherapy and Surgery. Principal Investigator: Norman Wolmar, MD. NSABP Foundation Inc. Available at: https://clinicaltrials.gov/ct2/show/ NCT01872975 Accessed 13 April 2018). Pre-ATNEC [Derby Hospitals NHS Foundation Trust. Pre-ATNECpreoperative tattooing of biopsied axillary node in breast cancer and correlation to sentinel lymph nodes in breast cancer patients (NCT03640819). www.clinicaltrials. gov. Accessed 16 June 2019].

Responder: SENTA [Kliniken Essen-Mitte. Prospective, multicenter, registry trial to evaluate utilization frequency and feasibility of targeted axillary dissection (TAD) after needle biopsy and clip placement in early breast cancer with clinically affected lymph nodes (NCT03102307). www.clinicaltrials.gov., 2019].

\section{Financial support and sponsorship}

None. 


\section{Conflicts of interest}

The authors have no conflicts of interest with regard to the contents of this review.

Other COIs: N.D.: MSD, Roche, Astrazeneca, Teva. T.K.: honorary from: Pfizer, Roche, Celgene. J.D.B.: Financial support: half salary by the Swedish Cancer Foundation with a Junior Clinical Investigator Award for 6 years. I.R.R.: I have no conflict of interest. M.L.G.: I have no conflict of interest and no financial support.

\section{REFERENCES AND RECOMIMENDED \\ READING}

Papers of particular interest, published within the annual period of review, have been highlighted as:

- of special interest

- of outstanding interest

1. Hunt $\mathrm{K}, \mathrm{Yi} M$, Mittendorf $\mathrm{E}$, et al. Sentinel lymph node surgery after neoadjuvant chemotherapy is accurate and reduces the need for axillary dissection in breast cancer patients. Ann Surg 2009; 250:558-566.

2. van der Heiden-van der Loo M, de Munck L, Sonke G, et al. Population based study on sentinel node biopsy before or after neoadjuvant chemotherapy in clinically node negative breast cancer patients: identification rate and influence on axillary treatment. Eur J Cancer 2015; $51: 915-921 ; 10$

3. Krag D, Anderson S, Julian T, et al. Sentinel-lymph-node resection compared with conventional axillary-lymph-node dissection in clinically node-negative patients with breast cancer: overall survival findings from the NSABP B-32 randomised phase 3 trial. Lancet Oncol 2010; 11:927-933.

4. Mamounas E, Brown A, Anderson S, et al. Sentinel node biopsy after neoadjuvant chemotherapy in breast cancer: results from National Surgical Adjuvant Breast and Bowel Project Protocol B-27. J Clin Oncol 2005; 23:2694-2702

5. van Deurzen C, Vriens B, Tian-Heijnan V, et al. Accuracy of sentinel node biopsy after neoadjuvant chemotherapy in breast cancer patients: a systematic review. Eur J Cancer 2009; 45:3120-3124.

6. Dosani M, Truong PT. Controversies in locoregional management of breast cancer with low volume $\mathrm{pNO}(\mathrm{i}+)$ and $\mathrm{pN} 1 \mathrm{mi}$ nodal disease. Expert Rev Anticancer Ther 2019; 19:803-810.

7. Carey LA. De-escalating and escalating systemic therapy in triple negative breast cancer. Breast 2017; 34(Suppl 1):S112-S115.

8. Jung $\mathrm{N}, \mathrm{Kim} \mathrm{HJ}$, Jung $\mathrm{JH}$, et al. Restaging the axilla after neo-adjuvant chemotherapy for breast cancer: predictive factors for residual metastatic lymph node disease with negative imaging findings. Breast J 2019; 25:196-201.

9. Pilewskie M, Morrow M. Margins in breast cancer: how much is enough? Cancer 2018; 124:1335-1341; Review.

10. Ditsch N, Bauerfeind I, Vodermaier A, et al. A retrospective investigation of women's experience with breast reconstruction after mastectomy. Arch Gynecol Obstet 2013; 287:555-561.

11. McCahill LE, Privette $A$, James $T$, et al. Quality measures for breast cancer surgery: initial validation of feasibility and assessment of variation among surgeons. Arch Surg 2009; 144:455-462; discussion 462-463.

12. Early Breast Cancer Trialists' Collaborative Group (EBCTCG). Long-term outcomes for neoadjuvant versus adjuvant chemotherapy in early breast cancer: meta-analysis of individual patient data from ten randomised trials. Lancet Oncol 2018; 19:27-39.

13. Choi J, Lews $A, \mathrm{Hu} J$, et al. Margins in breast-conserving surgery after neoadjuvant therapy. Ann Surg Oncol 2018; 25:3541-3547.

14. Ditsch $N$, Untch $M$, Thill $M$, et al. AGO recommendations for the diagnosis and treatment of patients with early breast cancer: update 2019. Breast Care (Basel) 2019; 14:224-245.

15. Negenborn VL, Young-Afat DA, Dikmans REG, et al. Quality of life and patient satisfaction after one-stage implant-based breast reconstruction with an acellular dermal matrix versus two-stage breast reconstruction (BRIOS): primary outcome of a randomised, controlled trial. Lancet Oncol 2018; 19:1205-1214.

16. Lohmander F, Lagergren J, Roy PG, et al. Implant based breast reconstruction with acellular dermal matrix: safety data from an open-label, multicenter, randomized, controlled trial in the setting of breast cancer treatment. Ann Surg 2019; 269:836-841.

17. Garcia-Etienne CA, Ferrari A, Della Valle A. Management of the axilla in patients with breast cancer and positive sentinel lymph node biopsy: an evidence-based update in an European breast center. Eur J Surg Oncol 2019. [Epub ahead of print]
18. Straver ME, Meijnen $P$, van Tienhoven $G$, et al. Sentinel node identification rate and nodal involvement in the EORTC 10981-22023 AMAROS trial. Ann Surg Oncol 2010; 17:1854-1861.

19. Giuliano $A E$, Hunt KK, Ballman KV, et al. Axillary dissection vs. no axillary dissection in women with invasive breast cancer and sentinel node metastasis: a randomized clinical trial. JAMA 2011; 305:569-575.

20. Rosenberger LH, Thomas SM, Plichta JK. Decreasing rates of axillary lymph node dissections over time: implications for surgical resident exposure and operative skills development. Am J Surg 2019; 218:786-791.

21. Poodt IGM, Spronk PER, Vugts G, et al. Trends on axillary surgery in nondistant metastatic breast cancer patients treated between 2011 and 2015. Ann Surg 2018; 268:1084-1090.

Article with summarizing of data of development of axillary procedures in the last decade. Significant decrease of completion axillary lymph node dissection as axillary staging between 2011 and 2015 .

22. Morrow $M$, Jagsi $R, M c L e o d ~ M C$, et al. Surgeon attitudes toward the omission -1 of axillary dissection in early breast cancer. JAMA Oncol 2018; 4:1511-1516. Obstet. 2013 Mar;287(3):555-561.

The American College of Surgeons Oncology Group (ACOSOG) Z0011 trial demonstrated clinicopathologic characteristics and nodal axillary burden between pure/mixed invasive lobular cancer and invasive ductal cancer patients. Results. Despite a higher proportion of sentinel lymph node (SLN) macrometastases and association with more positive nodes at axillary lymph node dissection (ALND), lobular histology does not predict the need for ALND. ALND is not indicated on the basis of histology among patients otherwise meeting Z0011 criteria.

23. Hennings $A$, Köpke $M$, Feißt $M$, et al. Which patient with sentinel-positive breast cancer after breast conservation will receive completion axillary lymph node dissection in routine clinical practice. Breast Cancer Res Treat 2019; 173:429-438.

24. Garcia-Etienne CA, Mansel RE, Tomatis M, et al., EUSOMA Working Group.

- Trends in axillary lymph node dissection for early-stage breast cancer in Europe: Impact of evidence on practice. Breast 2019; 45:89-96.

Presentation of current European recommendations of practice-changing for the surgical approach to the axilla in breast cancer. Data of 34 breast centers showed decrease of ALND since 2010 but wide differences among centers indicating the need of unified clinical guidelines.

25. Classe JM, Loaec C, Gimbergues $P$, et al. Sentinel lymph node biopsy without

- axillary lymphadenectomy after neoadjuvant chemotherapy is accurate and safe for selected patients: the GANEA 2 study. Breast Cancer Res Treat $2019 ; 173: 343-352$.

The study was designed to access accuracy and safety of SLN biopsy (SLNB) after neoadjuvant chemotherapy (NACT). Patients with no initial node involvement allows to safely avoid ALND. There was a low risk of ALND involvement after residual breast tumor and lympho-vascular invasion and initially positive lymph node involvement.

26. Morency D, Dumitra S, Parvez E, et al. Axillary lymph node ultrasound following - neoadjuvant chemotherapy in biopsy-proven node-positive breast cancer: results from the SN FNAC study. Ann Surg Oncol 2019. [Epub ahead of print]

Study with the following secondary endpoint: does axillary ultrasound predict axillary pathological complete response (pCR) and increase the accuracy of SLN? FNR decreased if ultrasound was added to SLNB but have also leed to unnecessary ALND in almost 8\%.

27. Galimberti V, Ribeiro Fontana SK, Maisonneuve P, et al. Sentinel node biopsy after neoadjuvant treatment in breast cancer: five year follow-up of patients with clinically node-negative disease before treatment. Eur J Surg Oncol 2016; 42:361-368

28. Martelli G, Miceli R, Folli $\mathrm{S}$, et al. Sentinel node biopsy after primary chemotherapy: long-term results of a retrospective study. Eur J Surg Oncol 2017; 43:2012-2020.

29. Schwentner L, Helms G, Neklijudova V, et al. Using ultrasound and palpation for predicting axillary lymph node status following neoadjuvant chemotherapy: results from the multicenter SENTINA trial. Breast 2017; 31:202-207.

30. Diego $E$, McAuliffe $P$, Soran $A$, et al. Axillary staging after neoadjuvant chemotherapy for breast cancer: a pilot study combining sentinel lymph node biopsy with radioactive seed localization of pretreatment positive axillary lymph nodes. Ann Surg Oncol 2016; 23:1549-1553.

31. Park S, Koo JS, Kim GM, et al. Feasibility of charcoal tattooing of cytologyproven metastatic axillary lymph node at diagnosis and sentinel lymph node biopsy after neoadjuvant chemotherapy in breast cancer patients. Cancer Res Treat 2018; 50:801-812.

32. Choy N, Lipson J, Porter $\mathrm{C}$, et al. Initial results with preoperative tattooing of biopsied axillary lymph nodes and correlation to sentinel lymph nodes in breast cancer patients. Ann Surg Oncol 2015; 22:377-382.

33. Siso C, de Torres J, Esgueva-Colmenarejo A, et al. Intraoperative ultrasound-

- guided excision of axillary clip in patients with node-positive breast cancer treated with neoadjuvant therapy (ILINA Trial): a new tool to guide the excision of the clipped node after neoadjuvant treatment. Ann Surg Oncol 2018; 25:784-791.

Ultrasound guided placement of a clip in the nodal-positive axillary lymph node prior to neoadjuvant chemotherapy. Forty-six patients had a clip placed in the positive node. In two (4.3\%) cases, the clip could not be seen prior to surgery and the patient underwent ALND; however, the clipped node was successfully removed by IOUS-guided excision in 44 patients. The ILINA trial is accurate in predicting axillary nodal status after NAT. 
34. Natsiopoulos I, Intzes $S$, Liappis $T$, et al. Axillary lymph node tattooing and targeted axillary dissection in breast cancer patients who presented as $\mathrm{cN}+$ before neoadjuvant chemotherapy and became cNO after treatment. Clin Breast Cancer 2019; 19:208-215.

35. Park S, Park JM, Cho JH, et al. Sentinel lymph node biopsy after neoadjuvant chemotherapy in patients with cytologically proven node-positive breast cancer at diagnosis. Ann Surg Oncol 2013; 20:2858-2865.

36. Caudle AS, Yang WT, Krishnamurthy S, et al. Improved axillary evaluation following neoadjuvant therapy for patients with node-positive breast cancer using selective evaluation of clipped nodes: implementation of targeted axillary dissection. J Clin Oncol 2016; 34:1072-1078.

37. Simons J, Van Nijnatten T, Van der Pol C, et al. Diagnostic accuracy of

- different surgical procedures for axillary staging after neoadjuvant systemic therapy in node-positive breast cancer: a systematic review and meta-analysis. Ann Surg 2019; 269:432-442.

The meta-analysis is significant for axillary staging by a combination procedure consisting of SLNB with excision of a pre-non specific type (NST) marked positive lymph node appears to be most accurate for axillary staging after NST in 20 unique studies with 2217 patients.

38. van Ninjatten TJA, Simons JM, Smidt ML, et al. A novel less-invasive approach for axillary staging after neoadjuvant chemotherapy in patients with axillary node-positive breast cancer by combining radioactive iodine seed localization in the axilla with the sentinel node procedure (RISAS): a Dutch prospective multicenter validation study. Clin Breast Cancer 2017; 17:399-402.

39. Alvarado $\mathrm{R}$, Yi M, Le-Petross $\mathrm{H}$, et al. The role for sentinel lymph node dissection after neoadjuvant chemotherapy in patients who present with node-positive breast cancer. Ann Surg Oncol 2012; 19:3177-3184.

40. Boileau JF, Poirier B, Basik M, et al. Sentinel node biopsy after neoadjuvant chemotherapy in biopsy-proven node-positive breast cancer: the SN FNAC study. J Clin Oncol 2015; 33:258-264.

41. Boughey JC, Suman VJ, Mittendorf EA, et al. Sentinel lymph node surgery after neoadjuvant chemotherapy in patients with node-positive breast cancer: the ACOSOG Z1071 (Alliance) clinical trial. JAMA 2013; 310:1455-1461.

42. Enokido $K$, Watanabe $C$, Nakamura $S$, et al. Sentinel lymph node biopsy after neoadjuvant chemotherapy in patients with an initial diagnosis of cytologyproven lymph node-positive breast cancer. Clin Breast Cancer 2016; 16:299-304.

43. Kuehn T, Bauerfeind I, Fehm T, et al. Sentinel-lymph-node biopsy in patients with breast cancer before and after neoadjuvant chemotherapy (SENTINA): a prospective, multicentre cohort study. Lancet Oncol 2013; 14:609-618.

44. Pinero-Madrona A, Escudero-Barea MJ, Fernandez-Robayna F, et al. Selective sentinel lymph node biopsy after neoadjuvant chemotherapy in breast cancer: results of the GEICAM 2005-07 study. Cir Esp 2015; 93:23-29.

45. Yu Y, Cui N, Li HY, et al. Sentinel lymph node biopsy after neoadjuvant chemotherapy for breast cancer: retrospective comparative evaluation of clinically axillary lymph node positive and negative patients, including those with axillary lymph node metastases confirmed by fine needle aspiration. BMC Cancer 2016; 16:808.

46. Zetterlund LH, Frisell J, Zouzos A, et al. Swedish prospective multicenter trial evaluating sentinel lymph node biopsy after neoadjuvant systemic therapy in clinically node-positive breast cancer. Breast Cancer Res Treat 2017; 163:103-110.

47. Donker M, Straver ME, Wesseling J, et al. Marking axillary lymph nodes with radioactive iodine seeds for axillary staging after neoadjuvant systemic treatment in breast cancer patients: the MARI procedure. Ann Surg 2015; 261:378-382.

48. Dashevsky BZ, Altman A, Abe H, et al. Lymph node wire localization postchemotherapy: towards improving the false negative sentinel lymph node biopsy rate in breast cancer patients. Clin Imaging 2018; 48:69-73.
49. Kim EY, Byon WS, Lee KH, et al. Feasibility of preoperative axillary lymph node

marking with a clip in breast cancer patients before neoadjuvant chemotherapy: a preliminary study. World J Surg 2018; 42:582-589.

Study with a small number of patients $(n=20)$ that demonstrates the technical feasibility of image-guided marker-clip placement on positive axillary lymph nodes before NAC and removal with SLNB (95.8\% identification rate). It can improve the accuracy of the residual disease evaluation on the axilla, especially in patients with negative SLNB results, and can identify candidates for limited axillary surgery after NAC.

50. Nguyen TT, Hieken TJ, Glazebrook KN, et al. Localizing the clipped node in patients with node-positive breast cancer treated with neoadjuvant chemotherapy: early learning experience and challenges. Ann Surg Oncol 2017; 24:3011-3016.

51. Plecha $D$, Bai $S$, Patterson $H$, et al. Improving the accuracy of axillary lymph node surgery in breast cancer with ultrasound-guided wire localization of biopsy proven metastatic lymph nodes. Ann Surg Oncol 2015; 22:4241-4246

52. Boughey JC, Ballman KV, Le-Petross $\mathrm{HT}$, et al. Identification and resection of clipped node decreases the false-negative rate of sentinel lymph node surgery in patients presenting with node-positive breast cancer (T0-T4, N1-N2) who receive neoadjuvant chemotherapy: results from ACOSOG Z1071 (Alliance). Ann Surg 2016; 263:802-808.

53. Hartmann $\mathrm{S}$, Reimer $\mathrm{T}$, Gerber $\mathrm{B}$, et al. Wire localization of clip-marked axillary lymph nodes in breast cancer patients treated with primary systemic therapy. Eur J Surg Oncol 2018; 44:1307-1311.

The prospective study of 30 patients evaluated preoperative ultrasound-guided wire localization of the clipped node in the clinical routine. All patients had a successful Clip-marking. Wire localization was possible in 24 cases (80\%) and cannot be recommended as standard procedure at the time.

54. Woods R, Camp M, Durr N, Harvey S. A review of options for localization of axillary lymph nodes in the treatment of invasive breast cancer. Acad Radiol 2019; 26:805-819.

55. Barron $A U$, Hoskin $T L$, Day $C N$, et al. Association of low nodal positivity rate

- among patients with ERBB2-positive or triple-negative breast cancer and breast pathologic complete response to neoadjuvant chemotherapy. JAMA Surg 2018; 153:1120-1126.

The retrospective study reviewed data from the NCDB 2010-2015 ( $n=30821)$ to evaluate nodal positivity rates in patients with cT1/cT2 N0 ERBB2-positive disease and triple negative breast cancer (TNBC) with a breast pCR after NACT. Highest rates of breast pCR were seen in ERBB2-positive disease (44.3\%) and TNBC (37.2\%) in contrast to hormone receptor-positive and ERBB2-negative patients (13.1\%). In patients with cNO ERBB2-positive disease or TNBC with breast $\mathrm{pCR}$, the nodal positivity rate was less than $2 \%$, which supports consideration of omission of axillary surgery in this subset of patients.

56. Tadros AB, Yeng WT, Krishnamurthy $S$, et al. Identification of patients with documented pathologic complete response in the breast after neoadjuvant chemotherapy for omission of axillary surgery. JAMA Surg 2017; 152:665-670

57. Samiei $S$, van Nijnatten TJA, Munck $L$, et al. Correlation between pathologic

- complete response in the breast and absence of axillary lymph node metastases after neoadjuvant systemic treatment. Ann Surg 2018. [Epub ahead of print]

Of 4084 patients selected from the Netherlands Cancer Registry showed a pCR in $24.1 \%$. In clinically node negative patients (cNO), 97.7\% with breast pCR had ypN0 compared with $71.6 \%$ without breast pCR $(P<0.001)$. In clinically node positive patients (cN1), 45.0\% with breast pCR had ypNo compared with $9.4 \%$ without breast $\mathrm{pCR}(P<0.001)$. Breast $\mathrm{pCR}$ achieved after NST is strongly correlated with ypNO in cNO patients, especially in HER2 + and triple negative subtypes. 